\title{
Design of the Higher Education System Based on Parallel Association Rules Algorithm
}

\author{
Yi Xu' ${ }^{1}$ and Feng Zhou $\mathbb{1 D}^{2}$ \\ ${ }^{1}$ Jiaxing Nanyang Polytechnic Institute, Jiaxing, Zhejiang 314000, China \\ ${ }^{2}$ Jiaxing Nanhu University, College of Information Engineering, Jiaxing, Zhejiang 314000, China
}

Correspondence should be addressed to Feng Zhou; usozf@zjxu.edu.cn

Received 20 December 2021; Revised 10 January 2022; Accepted 27 January 2022; Published 1 March 2022

Academic Editor: Zhiguo Qu

Copyright (c) 2022 Yi Xu and Feng Zhou. This is an open access article distributed under the Creative Commons Attribution License, which permits unrestricted use, distribution, and reproduction in any medium, provided the original work is properly cited.

\begin{abstract}
Aiming at improving the quality of online education, a higher education system based on parallel association rules algorithm is designed. In this study, the functional structure module of the system is divided into six modules, namely, the home page module, course module, teacher module, student module, administrator module, and personal center, so as to carry out a comprehensive treatment for students, teaching, and education resources. On the basis of mining the data association rules of the education system, the parallel association rules algorithm is used to identify and analyze the original data of the system, so as to fundamentally improve the ability of the system to process data and complete the personalized recommendation of educational resources and teaching evaluation feedback. Experiments show that the design system has greater information throughput, a short response time, and a resource utilization rate of more than $90 \%$. In addition, the audio teaching resource plays better, which proves that the system effectively achieves the design expectation.
\end{abstract}

\section{Introduction}

The purpose of higher education is to teach knowledge and cultivate comprehensive professional talents. Chinese degrees are divided into bachelor's degree, master's degree and doctor's degree $[1,2]$. Since reform and opening-up, our country higher education enterprise to obtain long-term development, remarkable achievements were made in reform. A socialist basic higher education system in various forms that meet the multilevel needs of national economic construction and social development has been initially formed. It has exported high-end talents to the development of the society and made contributions to our country's construction [3]. Firstly, vigorously developing high-tech production is a magic weapon for the economic take-off of many countries. China is taking the road of "using informatization to drive industrialization and industrialization to promote informatization." Higher education has become the leading force on this new road of industrialization. As the center of talent training, scientific research, and cultural communication, higher education has unique advantages in opening up new social industries. In recent years, the scientific research forces of colleges and universities have paid attention to the combination of scientific research and social needs, and the transformation of scientific research results into production technology is increasing year by year, constantly opening up new industries for the society [4]. Secondly, higher education can promote social and cultural construction. The profound cultural deposits, learning atmosphere, and value orientation of higher education exert a strong radiating effect on the society. The culture transmitted by higher education is a profound spiritual culture and a core culture of ideas and concepts. Hence, it has a profound and lasting influence on the society. In a sense, higher education has become the creative center of modern culture and the leader of social and cultural construction [5]. Thirdly, higher education can provide human resources for social development by cultivating talents. In the era of knowledge economy, knowledge and technology have become the decisive elements of economic development. Higher education plays an important role in cultivating talents needed for social development. Higher education plays an 
important role in the construction of lifelong education system, continuing education, building a learning-oriented society, learning-oriented region, and learning-oriented city. Finally, higher education can be a powerful driving force for the sustainable development of society. Education in a narrow sense refers to specially organized school education and in a broad sense refers to social practical activities that affect people's physical and mental development. Higher education cultivates high-quality talents through a number of educational activities, so that they have the knowledge and ability to promote the sustainable development of society, so as to promote the further development of society $[6,7]$.

It can be said that higher education promotes social development. Higher education is an important dependence and power source of social development, which cannot be separated from higher education. Therefore, in view of the unsatisfactory effect of traditional classroom teaching methods in reference [8], it is designed with the support of $5 \mathrm{G}$ wireless communication. Based on the actual needs, a new three-dimensional interactive teaching system is built by using the distributed architecture. According to the different use permissions of students and teachers, the teacher client module, login and account management module, course management module, and teaching interactive evaluation module are set up to comprehensively strengthen the function of the system. In the software module, 5G wireless communication technology is adopted to effectively remove the interfering information in the transmission channel of teaching information resources and complete power distribution, thus improving the anti-interference performance and resource update rate of the system [9]. In view of the limitations of traditional education methods in time and space, the Struts + Spring + Hiber-Nate framework popular in Java technology is used to build a professional and integrated modern online education system. Based on $\mathrm{O} 2 \mathrm{O}$ model, the system realizes the organic combination of online and offline education and provides an online education management platform for teachers and a community platform for online communication for students. According to reference [10], teaching resources can only be shared with a single client because of the single transmission in the traditional distance education system. To realize the sharing of teaching resources, the university distance education system based on ASP technology is designed under the background of internet. Based on the optimization of the sharing server and the structure design of the sharing device, the hardware environment of the system is designed, and the software of the system is designed based on the system database and the login interface. However, in practical application, it is found that the ideal degree of information throughput, resource utilization, and response speed of the education system is low. To solve this problem, this paper designs a higher education system based on parallel association rules algorithm.

\section{Higher Education System Design}

2.1. Functional Structure Design of the System. According to the functional requirements of the system, the functional result modules of the higher education system are set as shown in Figure 1.
As shown in Figure 1, the functional structure modules of higher education system mainly include the home page module, course module, teacher module, student module, administrator module, and personal center. In the construction of the system, the interface is designed using DreamWeaver CS6 to complete the development, and after the development of the design code, it is transferred to Visual Studio, the use of which controls to replace the page's need to update the label. Enhance the security of the guarantee system, and give the interface design code of the system. The functional code and background code are written separately to minimize the code type in the page. If one needs to modify the code, just click the code file to modify.

System data affects the stability and the ease-of-use of the platform, and it is an important basis for system operation $[11,12]$. The common variables within the system are time and the latest preview of teaching resources, which are constantly changing with access status. In the design of the system, the quantity of this aspect is regarded as a variable, and the data in the page will be changed with the update of the data.

\subsection{System Modular Design}

2.2.1. Home Page Module. The home page has four submodules of personalized recommendation, learning discussion, learning check-in, and voting functions. Students, teachers, and system administrators can complete the system-related content view, check-in, and related learning content discussion, and they can participate in the questionnaire survey using the home page function. Among them, in the home page of the system, various types of course materials will be displayed. Through the personalized recommendation submodule, students can recommend learning resources that meet their needs. The essence of the learning and discussion submodule is a forum that is mainly responsible for the communication between teachers and students. At the same time, teachers can timely solve problems for students. When students enter the system for learning, they need to sign in the learning check-in submodule to confirm that they have completed the day's learning. The submodule of voting function supports teaching evaluation and teaching feedback.

2.2.2. Course Module. The module includes teaching case, case problem sets, video reference books, and courses by four modules, the users can view the ideological and political teaching content and teaching plan at any time, extract the preview classroom learning content, or leave a message by viewing the teaching directory.

The curriculum module is one of the core modules of the system, which is mainly responsible for the improvement of higher education materials. For mobile terminal users, simple and atmospheric modules are required $[13,14]$. On the left side of the interface, different types of course materials and table of contents are set. On the right side of the interface, buttons for teaching plans and exercises are set. After the user clicks the 


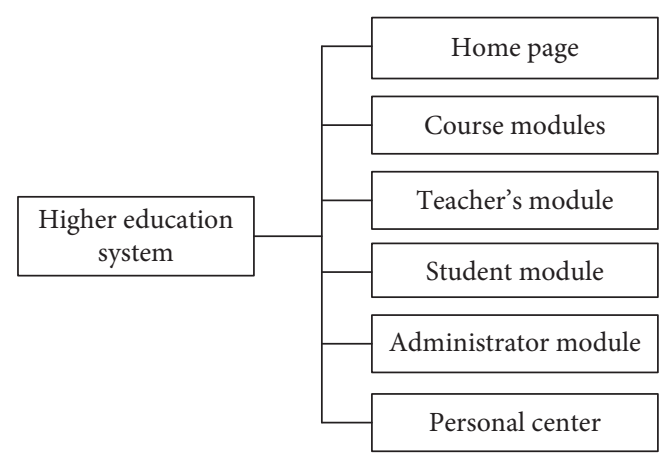

Figure 1: System functional structure division diagram.

selected course category, the user enters the current module. The user selects different interfaces according to the information he/ she needs. At the same time, the course module provides users with viewing and downloading functions.

When the system users find learning resources to play, the system will automatically transfer to the course module. During the video playback, buttons are displayed to control the playback progress and sound. In addition, the first view embedded in the system is the introduction view of the current course, which comprehensively shows the characteristics of the current subject to students. When the introduction button is clicked, the system interface will display the introduction of various courses, suitable groups, and introduction of teachers' teams $[15,16]$.

Fully reflect the personalized of the system, the module has also designed the function of bullet screen view, mainly to realize the online communication of users and comprehensively improve the learning interest of college students.

The system divides the difficulty degree of course resources into five grades. Using the five-point scale can not only facilitate resource management but also achieve scientific classification of resource grades. The evaluation of curriculum resources should not only be based on evaluation results but also refer to teachers' opinions.

Suppose $D=\left\{D_{1}, D_{2}, D_{3}, D_{4}, D_{5}\right\}$ represents the difficulty level of resources corresponding to different difficulty levels. $D_{1}$ is very simple, and the weight is -2 . $D_{2}$ corresponds to simple, and the weight is $-1 . D_{3}$ stands for medium, and it has a weight of $0 . D_{4}$ means difficult, and the weight is $1 . D_{5}$ is very difficult to characterize. It has a weight of 2 .

The difficulty of class $j$ course resource evaluation is expressed as follows:

$$
D_{j}(\text { voting })=\sum_{j=1}^{5} \frac{n_{k j}}{N_{k j}},
$$

where $D$ (voting) represents the weighted average of the difficulty of class $j$ course resources after students complete evaluation, $n_{k j}$ represents the number of students of class $j$ course resources determined as the difficulty of $k, N_{k j}$ is the total number of students participating in the evaluation of class $j$ course resources, and its expression is as follows:

$$
N_{k j}=\sum_{j=1}^{5} n_{k j} .
$$

The difficulty adjustment process of learning resources can be expressed as follows:

$$
D(\text { tuned })=w \times D(\text { initial })+(1-w) \times D(\text { voting }),
$$

where $D$ (initial) is the initial difficulty value of class $j$ course resources by teachers, $D$ (voting) is the average difficulty value that students think of class $j$ course resources, $D$ (tuned) is the adjusted difficulty value of class $j$ course resources, and $w$ is an adjustable weight.

By the assessment of students' learning ability and the adjustment of the difficulty of resources, the connection between students and the difficulty of course resources is established to meet the current cognitive level of students and achieve the purpose of self-adaptation of teaching resources. This process not only reflects the effective interaction between teachers and students but also helps students improve the teaching effect $[17,18]$.

2.2.3. Teacher Module. Teacher account is divided into publishing, modifying introduction, course introduction, uploading courseware, assigning homework, and so on. Specific functions are shown in Figure 2.

When designing the teacher module, this paper designs the teacher account authority and implementation logic based on the relevant functional requirements of the course. Registration is also required. The administrator will verify the registration information according to offline information, and teachers can log in to the system after verification.

2.2.4. Student Module. The main functions of the student module are shown in Figure 3.

After logging into the personal account, students can view the teacher and course introduction, select courses based on the introduction content, and download the selected course resources. In the learning process, the online note-taking function can be used to record the knowledge points that are not fully mastered and put forward to the teacher in the after-class Q\&A session to achieve teacherstudent interaction $[19,20]$.

Aiming at the characteristics of abundant teaching resources in the system, this paper systematically designs an adaptive guidance mechanism that adapts to the individualized learning needs of students. To achieve the purpose of self-adaptation, students need to do a predictive test when logging in the system for the first time, which tests students' cognitive ability by showing them the knowledge points of various difficulty levels.

Assume that the test result is expressed as $W=\left\{W_{1}, W_{2}, \ldots, W_{i}, \ldots, W_{m}\right\}$, where $W_{i}$ is the feedback value of question $i$, which is either 1 or $0 . W_{i}=1$ means correct answer to question $\mathrm{I}$, and $W_{i}=0$ means the wrong answer to the same. The relationship between cognitive status and ability value can be expressed by maximum likelihood estimation.

If all the answering processes of students are independent of each other, it can be considered that the evaluation results of the ability value are only related to cognitive level and difficulty degree of the question [21, 22]. Under the above assumptions, the cognitive level assessment process can be expressed as follows: 


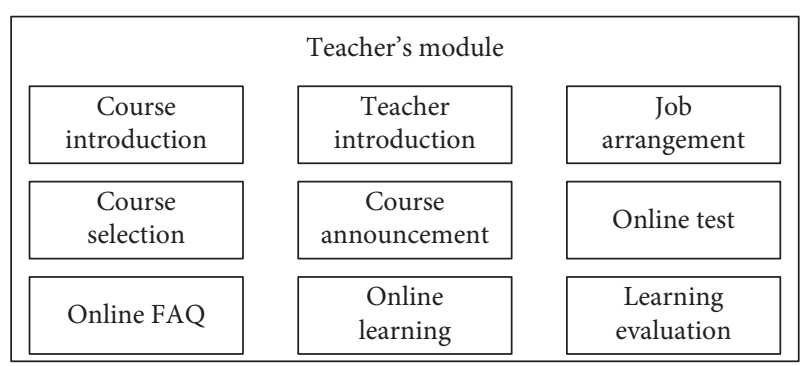

FIGURE 2: Teacher module function diagram.

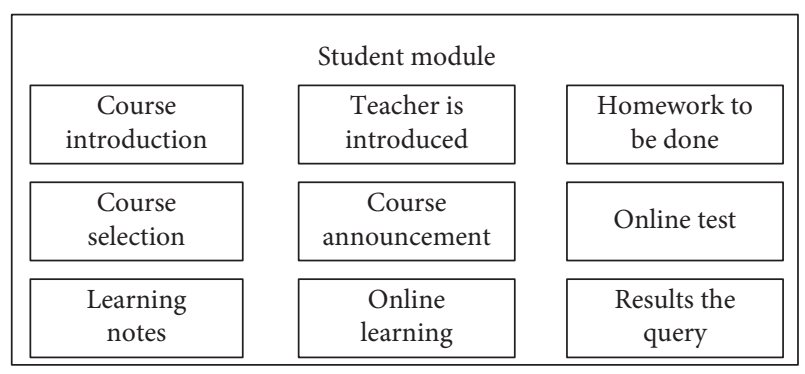

Figure 3: Student module function diagram.

$$
L(W \mid f)=\prod_{i=1}^{m}=P_{i}(f)^{W_{i}} Q_{i}(f)^{1-W_{i}}
$$

where the likelihood function $f$ shows the relationship between students' cognitive level and test results. Since the likelihood estimation is positively correlated with the ability value, the ability value can be obtained by taking the derivative of $f . P_{i}(f)$ and $Q_{i}(f)$, respectively, represent the probability of correct or wrong answer on question $i$ when the student's cognitive level is $\theta$.

Theoretically, both $P_{i}(f)$ and $Q_{i}(f)$ are functions to show cognitive level and teaching resource level. Hence, their values exert certain influence on the likelihood function through $P_{i}(f)$ and $Q_{i}(f)$. The comprehensive analysis of derivative relationship shows that the function value increases with the increase of capability value [23-25]. It is assumed that the range of likelihood function value is $[-3,3]$. If students' ability value is -3 , their cognitive level is considered to be weak. When the ability value is 0 , the cognitive level is average. When the ability value is 3 , it indicates that students have a strong cognitive level.

Based on the above process system, the reasonable evaluation of students' cognitive level can be completed. Next, it is necessary to adjust the difficulty of knowledge points so that students can match the most suitable learning resources and improve learning efficiency.

2.2.5. Administrator Module. In this module, the system administrator can maintain the system, audit all kinds of teaching resources, approve user registration, etc. In addition, the administrator can also query and modify the information in the system according to the needs.
2.2.6. Personal Center. The personal center module has two submodules of login and registration, which can modify user personal information at any time. The client of the system is not a single school but a statistical integration of all teaching clients. System users need to fill in complete registration information during account registration.

2.3. System Software Running Program Design. On the basis of the above modular design of the system, this study completed the design of the system software running program based on the parallel association rule algorithm. It mainly used the parallel association rule algorithm to preprocess the system data, and it completed the resource recommendation and teaching evaluation feedback.

\subsubsection{Based on the Parallel Association Rule Algorithm} Processing System Basic Data. Parallel association rules are the most commonly used methods in data mining and processing [26-28], which includes the following contents:

(1) Item and Item Set. The values of each field in the dataset are inconsistent, assuming that each value is assigned an item. The set of terms is used to describe terms, and the set of $g$ terms is represented by $g+$ term set.

Item set $I=\left\{I_{1}, I_{2}, I_{3}, \ldots, I_{g}\right\}$ because there are $g$ items in the item set, and $I$ is called $g+$ item set.

(2) Confidence. Confidence is a feature of association rules. The confidence of association rules $X \Longrightarrow Y$ describes that the database may contain $X$ and $Y$, which can be expressed as $\operatorname{Conf}(X \Longrightarrow Y)$. The calculation method is as follows:

$$
\operatorname{Conf}(X \Longrightarrow Y)=\frac{\operatorname{Support}(X \cup Y)}{\operatorname{Support}(X)}
$$

Therefore, the user sets the minimum confidence, which is expressed as follows: $\min \_$Conf $(X \Longrightarrow Y)$.

(3) Number and Degree of Support [29, 30]. The supported number represents the number of occurrences of item set $I$ in the database, represented by $\varphi_{I}$. Support is the ratio of item set support number $\lambda_{x}$ to the total number of things database, which is expressed as Support (I), and its calculation process is as follows:

$$
\operatorname{Support}(I)=\frac{\varphi_{I}}{|H|}
$$

where the total number of databases is represented by $|H|$.

(4) Association Rules. Association rule is the implied expression describing $X \Longrightarrow Y$, where $X \subseteq I, Y \subseteq I$, and $X \cap Y=\varnothing$. This rule is used to describe the implicit relationship between databases $[31,32]$. The front component is $X$, and the back component is $Y$. The rules that meet the following requirements are 
called strong association rules. Otherwise, they are called weak association rules.

(a) Confidence degree $\operatorname{Conf}(X \Longrightarrow Y)$ is not less than the specified minimum confidence $\min { }_{-} \operatorname{Conf}(X \Longrightarrow Y)$, i.e., things with Conf $(X \Longrightarrow Y)$ in the database include when $X$ also includes $Y$.

(b) Support Support $(X \cup Y)$ is not less than the specified minimum support min_Conf $(X \Longrightarrow Y)$, i.e., the number of things with Support $(X \cup Y) \times|H|$ in the database contains both $X$ and $Y$.

Parallel association rules mainly meet the following two conditions:

(1) Search for frequent item sets: find item sets whose support is not less than the minimum support specified by users $[33,34]$.

(2) Establishing parallel association rules: it means that the association rules can be constructed by combining the frequently item sets searched above, and the confidence degree of the association rules is required to be greater than or equal to the minimum confidence degree set by a user. If frequent item set $P$ exists and all nonempty item sets of $P$ are detected, respectively, then association rule $I \Longrightarrow P-I$ is obtained, and the confidence degree of this association rule is obtained. Association rules whose confidence degree is greater than or equal to the minimum are retained, and other unqualified ones are deleted. According to the characteristics of association rules, the forward detection of association rules can be simplified to the detection of the largest subset $P$, and the smaller subset can be detected only when the requirements are met.

The process of simplifying mining parallel association rules includes data preprocessing, discovering frequent item sets, and generating association rules and rule interpretation $[35,36]$. Therefore, in the higher education system designed in this study, the specific steps for generating parallel association rules of system basic data are as follows:

Step 1: work out all nonempty item sets $X_{I}$ of the frequent item sets $X$ of the education system data.

Step 2: by scanning the data set, calculate the support degree of all nonempty item sets, namely,

$$
\operatorname{Support}\left(X_{I}\right)=\frac{\left(X_{I}\right)}{P \times \text { count }} \text {. }
$$

Step 3: scan all data sets, and calculate the confidence $X_{I} \Longrightarrow R$ of the parallel association rule between the item set $X_{I}$ and original data $R$, which is the ratio of the record times of the data containing $X_{I}$ and $R$ to the record times of the data containing only $X_{I}$ in the combined data set. The process is as follows:

$$
\text { Confindence }\left(X_{I} \Longrightarrow R\right)=\frac{\left(X_{I}, R\right)}{X_{I} \times \operatorname{coun}} \text {. }
$$

Step 4: arrange the association rules that meet the minimum confidence condition in the descending order, and the result of this arrangement is the standard of optimal educational data.

By mining the parallelized association rules among the data of the education system above, the original data of the system can be identified and analyzed, and the ability of the data processing of the system can be fundamentally improved.

2.3.2. Educational Resources Recommendation. In this study, personalized recommendation tasks for educational resources are completed on Spark platform. The specific recommendation process is shown in Figure 4:

Step 1: Firstly, the data analysis system analyzes the user's initial score and score matrix and then calculates the similarity between users through vector cosine algorithm. The similarity between user $\mathrm{a}$ and user $\mathrm{b}$ is as follows

$$
S(a, b)=\frac{Y(a) \cap Y(b)}{Y(a) Y(b)} .
$$

Equivalence principle can be obtained, and the expression formula of similarity between teaching resources $P$ and $Q$ is as follows:

$$
S(P, Q)=\frac{Y(P) \cap Y(Q)}{Y(P) Y(Q)} .
$$

Step 2: set the loss function and substitute in the above similarity calculation results to obtain the following:

$$
f(u, v)=\left(\frac{S(a, b) g_{u}}{S(a, b)+1}\right)^{2}+\left(\frac{S(P, Q) g_{v}}{S(P, Q)+1}\right)^{2},
$$

where $g_{u}$ represents $N$ nearest neighbors of user $u$, and $g_{v}$ represents $N$ nearest neighbors of teaching resources.

Step 3: perform the iterative calculation of the minimum function in Step 2 to obtain the matrix of $u$ and $v$ statistics. Then, based on the iterative calculation, complete the personalized recommendation of educational resources according to the similarity between resources and users.

2.3.3. Teaching Evaluation and Feedback. Through the evaluation and record of students'achievement and teaching, the real teaching evaluation and feedback results can be obtained by comprehensive analysis of students'learning.

The relevant data used in teaching evaluation and feedback mainly include basic teaching information and student performance data. 


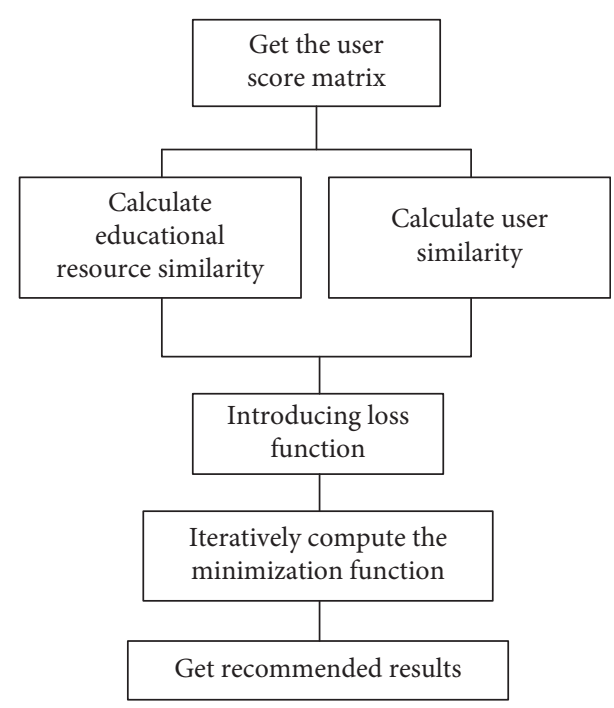

Figure 4: Personalized recommendation process for educational resources.

(1) Basic Information. Teacher basic information: teacher is represented by $T$ in combination, and its expression is as follows:

$$
T=\left\{t \mid t \in \prod_{i=1}^{N} \operatorname{dom}(A)\right\},
$$

where $A$ is the attribute set of teacher information, including teacher identity, professional title, educational background, etc., and $\operatorname{dom}(A)$ is the attribute component field.

Basic information of students: $S$ is the set of students, and the formula is as follows:

$$
S=\left\{s \mid s \in \prod_{i=1}^{N} \operatorname{dom}(B)\right\},
$$

where $B$ is the set of student information attributes, including student information, gender, telephone number, etc.

Course content: $C$ is the course set, and the formula is as follows:

$$
C=\left\{c \mid c \in \prod_{i=1}^{N} \operatorname{dom}\left(C^{\prime}\right)\right\} .
$$

Here, $C^{\prime}$ includes course number and course name.

(2) Student Performance Information. Grade type information is represented by $R$, and the expression is as follows:

$$
R=\left\{r \mid r \in \prod_{i=1}^{N} \operatorname{dom}(D)\right\},
$$

where $D$ is the information set related to the score type, including the name of the test subject, weight, and remarks.

Score information: $F$ is the set, which is as follows:

$$
F=\left\{f \mid f \in \prod_{i=1}^{N} \operatorname{dom}(G)\right\},
$$

where $G$ includes student number, name, total score, and time.

On this basis, obtain the student achievement information. Student scores can be collected in a number of ways. In this paper, the following methods are used for collection and evaluation:

(1) Classroom status information: this information shows the mood and attitude in learning. By the method of teacher evaluation to show the students' participation and preparation, at the same time, the data is retained.

(2) Homework information: this information is one of the most intuitive data for grade evaluation. The system can record the speed of writing homework and the situation of wrong questions, and it can directly show the degree of learning students. At the same time, the system reduces the amount of teachers' tasks. They only need to check the subjective questions, after which the review results are automatically stored in the database, which the students and teachers can view.

(3) Test information: test information is similar to homework information, which can directly reflect the learning situation and play a good feedback role. Students complete the exam using the online test submodule, which is corrected by the instructor and recorded in the database.

(4) Social performance information: this information is the embodiment of students' comprehensive quality. The evaluation results of students' social performance can show the results of students' comprehensive development. Social performance is a way to examine students' comprehensive ability. After setting the relevant content of social performance in the system, the score can be directly matched with the set.

All the data of teaching evaluation and feedback of higher education system can be obtained using the above methods, and the teaching evaluation and feedback can be completed using the processing process shown in Figure 5.

The page of higher education system based on parallel association rule algorithm is shown in Figure 6.

\section{Experiment and Analysis}

To verify the feasibility of the system designed in this paper, the subsequent experimental verification process is designed.

3.1. Preparation. In the experiment, four servers of different types, two switches, and several computers with the same configuration were used to simulate the higher teaching 


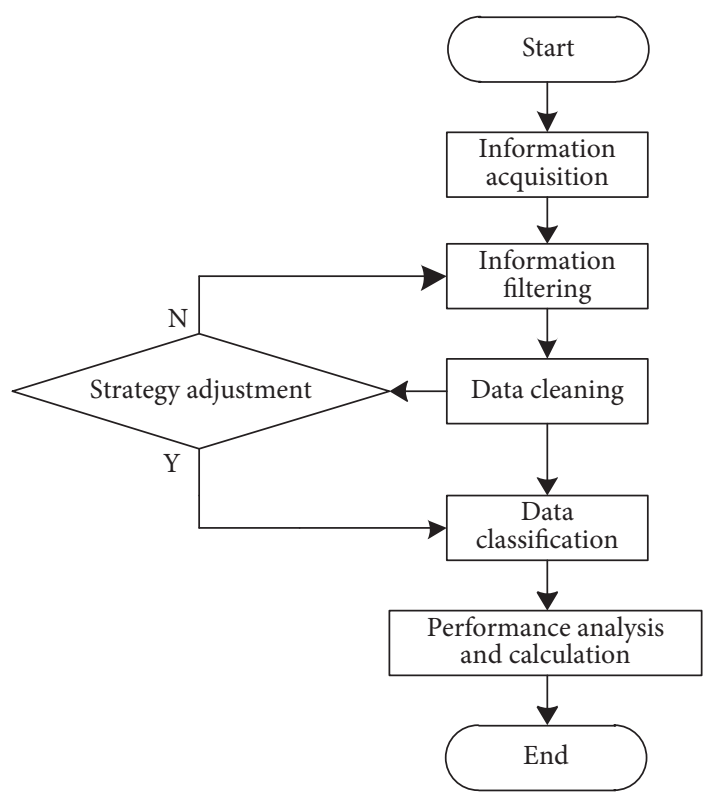

FIgURE 5: Systematic teaching evaluation and feedback flow chart.

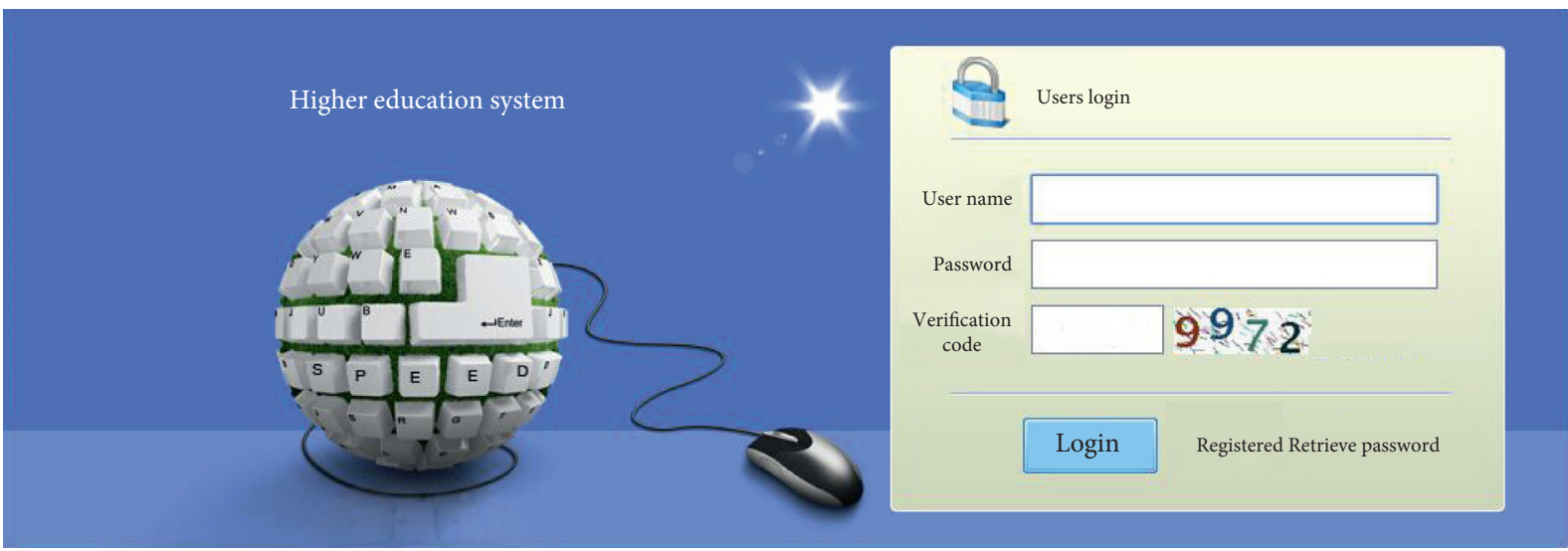

Figure 6: Higher education system page.

operating environment by constructing a topology structure. The topological structure of the established teaching system is shown in Figure 7:

Client: the operating system is Window10, the memory is $8 \mathrm{~GB}$, and the disk is $500 \mathrm{~GB}$

Data server: Linux operating system, $8 \mathrm{~GB}$ memory, and 1 TB hard disk

Application Server: Windows Server 2020, 25 GB memory, and 2 TB hard disk

The experiment was carried out on the MATLAB platform. According to the experimental environment designed above, the practical application performance of the higher education system designed in this study based on the parallel association rule algorithm was verified from the perspectives of information throughput, resource utilization, system response sample, and audio resource playback effect.

To avoid too many single experimental results, the EDUCATION system based on $\mathrm{O} 2 \mathrm{O}$ in reference [9] and the education system based on ASP technology in reference [10] were compared with complete performance verification together with the system in this paper.

3.2. Results and Analysis. Firstly, design the experimental index as the information throughput and analyze the application effect of the system, as shown in Figure 8 .

The analysis of the results shown in Figure 8 shows that the information throughput of the three systems is increasing in unit time. The information throughput of the education system based on $\mathrm{O} 2 \mathrm{O}$ varies between $800 \mathrm{~KB}-1600 \mathrm{~KB}$, the information throughput of the education system based on ASP technology varies between $1300 \mathrm{~KB}-1800 \mathrm{~KB}$, and the information throughput of the system in this paper varies between $1600 \mathrm{~KB}-2500 \mathrm{~KB}$. In contrast, the information throughput of the system in this paper is larger. It proves that it can successfully transmit the largest amount of data in the same time. It shows that the 


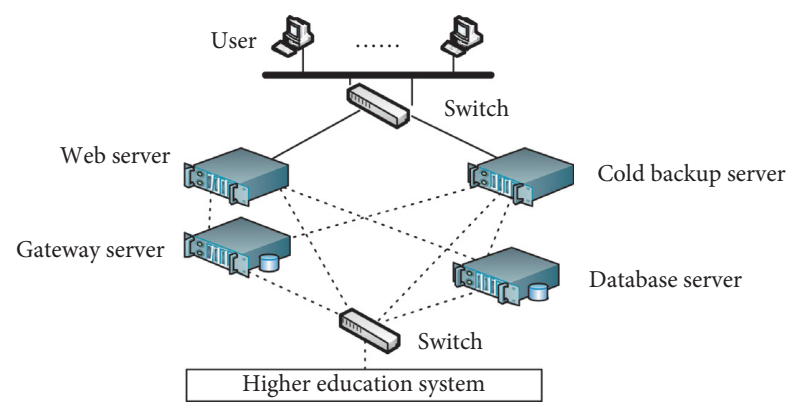

FIGURE 7: Teaching system topological structure diagram.

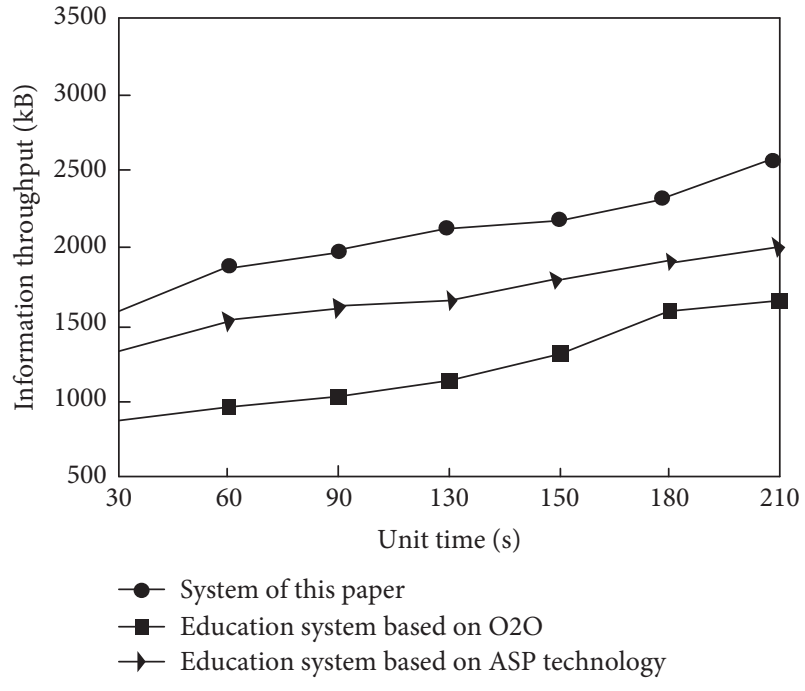

Figure 8: System information throughput test results.

software function of this system is powerful and the program efficiency is high.

Secondly, design the experiment index for resource utilization and analyze the application effect of the system, as shown in Figure 9.

The analysis of the results shown in Figure 8 shows that the resource utilization of different systems is changing with the increase of the number of physical nodes. In the whole test process, the resource utilization rate of the education system based on $\mathrm{O} 2 \mathrm{O}$ is between $82 \%$ and $86 \%$ and that based on ASP technology is between $68 \%$ and $86 \%$. However, the resource utilization rate of the system in this paper always remains above $90 \%$, and there is no imbalance phenomenon of the high utilization rate of a few nodes and low utilization rate of the rest nodes. It is proved that the system can integrate resources quickly without local overload. In contrast, the resource utilization value of the two traditional systems is always lower than that of the system in this paper.

Then, the system response specimens were used as indicators to compare the application effects of different systems, as shown in Figure 10.

According to the results shown in Figure 10, different systems have different response times. Among them, the

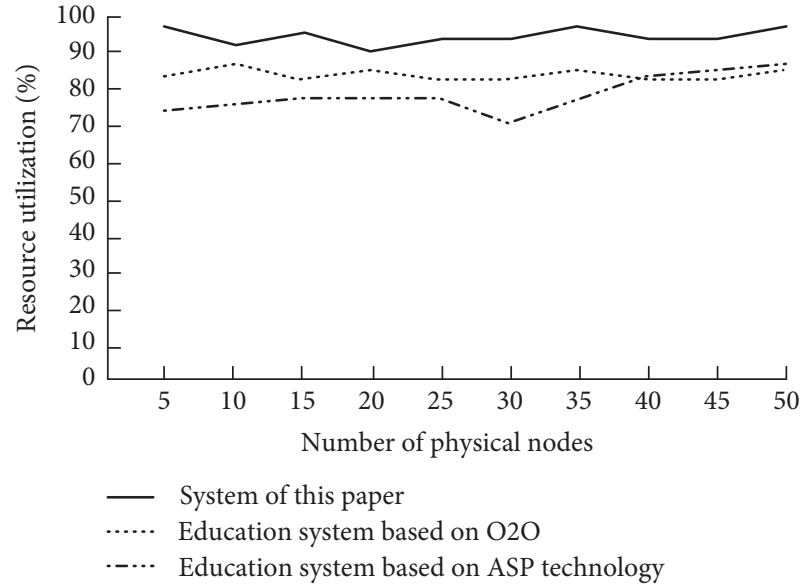

FIgURE 9: Resource utilization test results for different systems.

test response time is the highest among the five responses. The test response time of the $\mathrm{O} 2 \mathrm{O}$-based education system is $5.1 \mathrm{~s}$, and the test response time of the education system based on ASP technology is as high as $8.0 \mathrm{~s}$, while the response time of the design system is only $3.8 \mathrm{~s}$, which is lower than the other two systems. Therefore, the response speed of the system in this paper is faster.

Finally, test the audio resource playing effect of different systems. A piece of teaching audio with the same memory size and teaching content is played in the three systems. The experimental results of the audio waveform obtained in the audio extractor for the three systems are shown in Figures 11-13.

As can be seen from the waveform results shown in Figures 11-13, after the application of $\mathrm{O} 2 \mathrm{O}$ education system, there is waveform loss between $3 \mathrm{~s}$ to $4 \mathrm{~s}$ of audio. After the application of education system based on ASP technology, there is a loss of waveform between $2 \mathrm{~s}$ to $3 \mathrm{~s}$ and $4 \mathrm{~s}$ to $5 \mathrm{~s}$ of audio. After the application of the system in this paper, there is no audio data loss in the process of playing teaching audio, which proves that the system in this paper can play teaching audio resources completely.

To sum up, the higher education system based on parallel association rule algorithm designed in this study has the application advantages of large information 


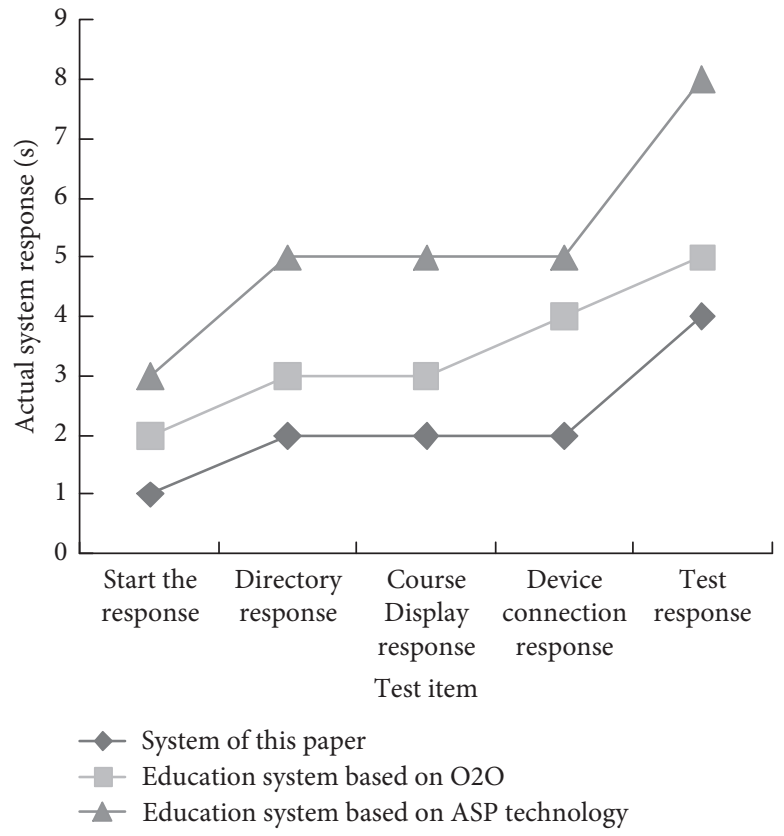

FIGURE 10: Response time test results for different systems.

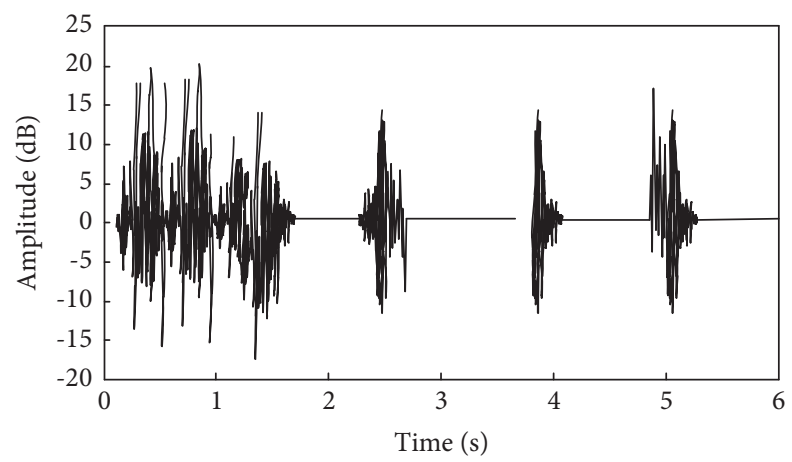

Figure 11: Based on $\mathrm{O} 2 \mathrm{O}$ education system playing audio waveform results.

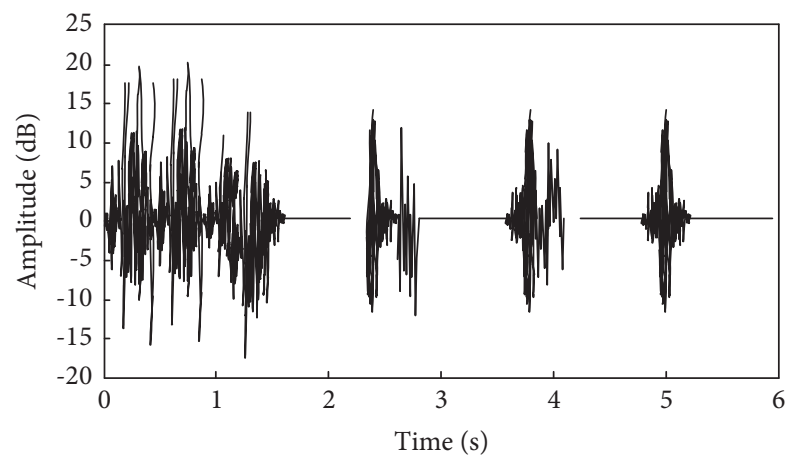

FIGURE 12: Waveform results of audio playback in education system based on ASP technology. 


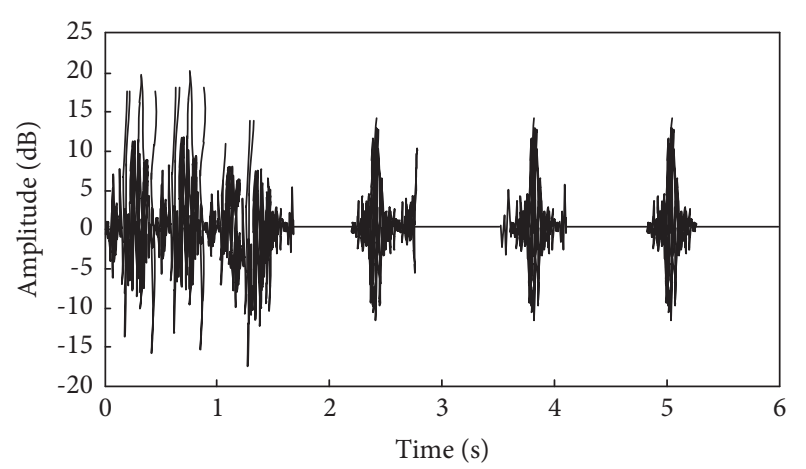

FIGURE 13: This paper presents the waveform results of playing audio in the system.

throughput, high resource utilization, and fast response speed, and the playback effect of audio teaching resources is better.

\section{Conclusion}

This paper introduces parallel association rule algorithm to design a higher education system. Firstly, it divides several functional modules of the system, and then it identifies and analyzes the original data of the system using the parallel association rule algorithm to complete the personalized recommendation of educational resources and teaching evaluation feedback. According to the experimental results, the information throughput of the system in this paper is larger, the resource utilization rate is always above $90 \%$, and the resource utilization rate is higher. In addition, the test response time of the system in this paper is only $3.8 \mathrm{~s}$, and the response efficiency is higher. Moreover, after the application of the system in this paper, there is no audio data loss in the process of playing teaching audio. It is proved that the system in this paper can play teaching audio resources completely, and the practical application effect is better.

\section{Data Availability}

The raw data supporting the conclusions of this article will be made available by the authors without undue reservation.

\section{Conflicts of Interest}

The authors declare that they have no conflicts of interest regarding this work.

\section{Acknowledgments}

This work was supported by industry-education integration practice project in the colleges of Zhejiang Provincial Department of Education, "Exploration on Collaborative Construction of EMS Talent Base Based on the Integration of Industry and Education," and Research Project on Teaching Reform of Higher Education in Zhejiang Province, "Exploration and Practice of Craftsman Talent Training Mode of Mechatronics Technology Specialty under the Background of "Dual System" Localization-Take "BHS Dual System" Class as an Example" (jg20160388).

\section{References}

[1] M. Yuan and C. Li, "Research on global higher education quality based on BP neural network and analytic hierarchy process," Journal of Computer and Communications, vol. 9, no. 6, pp. 158-173, 2021.

[2] Z. He, "Ecological interpretation of higher education system," Heilongjiang Researches on Higher Education, vol. 30, no. 12, pp. 1-4, 2012.

[3] Y. Xiang and J. Huang, "Supply-side and demand-side evolution analysis of higher education system," Course Education Research, vol. 5, no. 10, pp. 177-178, 2018.

[4] P. K. Madeshia and R. Sharma, "Higher education system \& quality teaching," Journal of Critical Reviews, vol. 7, no. 7, pp. 1216-1220, 2020.

[5] M. Su and B. Chen, "Co-evolutionary analysis of artificial intelligence and higher education," Higher Education Exploration, vol. 9, pp. 14-19, 2019.

[6] G. Fang, R. Li, and Z. Li, "The modernization of EU higher education Governance: development process, policy logic and practical characteristics," Modern Education Management, vol. 10, pp. 113-120, 2021.

[7] Z. Fu, "Analysis on the development of higher education management innovation under the background of cyberspace," Journal of Contemporary Educational Research, vol. 3, no. 6, pp. 618-625, 2019.

[8] X. Lu, F. Liu, and L. Wang, "Design of university stereo classroom interactive teaching system based on $5 \mathrm{G}$ wireless communication," Modern Electronics Technique, vol. 44, no. 12 , pp. 21-24, 2021.

[9] M. Xian, "Research and Implementation of education system based on O2O model," Automation \& Instrumentation, vol. 9, pp. 202-204, 2018.

[10] L. I. Xiang, "Design of university distance education system based on ASP technology under background of the Internet," Modern Electronics Technique, vol. 43, no. 23, pp. 178-181, 2020.

[11] G. Barbato and M. Turri, "What do positioning paths of universities tell about the diversity of higher education systems? an exploratory study," Studies in Higher Education, vol. 45, no. 9, pp. 1919-1932, 2020.

[12] M. Salas-Velasco, "The technical efficiency performance of the higher education systems based on data envelopment analysis with an illustration for the Spanish case," Educational Research for Policy and Practice, vol. 19, no. 3-4, pp. 159-180, 2020.

[13] M. Liliana, O. Clipa, and K. Tzafilkou, "The development and validation of a scale to measure university teachers' attitude towards ethical use of information technology for a sustainable education," Sustainability, vol. 12, no. 15, pp. 205-217, 2020.

[14] M. Sun, "Transformation of Chinese higher continuing education system-analysis based on parsons' AGIL model," Continue Education Research, vol. 1, pp. 9-12, 2020.

[15] S. Hong and L. Zhang, "The realistic context and evolution logic of higher education internationalization research: an empirical study based on WOS database," Higher Education Exploration, vol. 5, pp. 120-128, 2020.

[16] S. Mustica, "Communication and didactics, the difficult but needful symbiosis in higher education systems," Geopolitical, Social Security and Freedom Journal, vol. 2, no. 2, pp. 1-13, 2019.

[17] W. E. Tadesse, "Decolonising a higher education system which has never been colonised"," Educational Philosophy and Theory, vol. 53, no. 9, pp. 894-906, 2021. 
[18] Y. G. Tillayevna and N. M. Sunnatovna, "Further training of teachers as a condition for improving the quality of education," Academicia: An International Multidisciplinary Research Journal, vol. 10, no. 5, pp. 1931-1936, 2020.

[19] Q. Hu, W. Wu, F. Guang, T. Pan, Z. Chen, and K. Qiu, "Research on the key technology and practice of higher education teaching evaluation in the AI era," Education Research, vol. 27, no. 5, pp. 15-23, 2021.

[20] L. Yuan, K. Cai, L. I. Fang, and F. Zhang, "System and evolution mechanism of "internet + high education"," Meitan Higher Education, vol. 37, no. 6, pp. 55-59, 2019.

[21] M. Wu, Z. Gao, L. Hou, and J. Fan, "Innovation performance evaluation of regional higher education in China," Statistics and Decision, vol. 37, no. 15, pp. 170-174, 2021.

[22] L. I. Guonian, X. Tan, and H. Xiao, "Research on knowledge fusion model of decision support system for higher vocational education," Education and Vocation, vol. 10, pp. 84-91, 2020.

[23] D. Xu and L. Li, "Research on parallelization of association rules mining algorithm based on spark," Computer Technology and Development, vol. 29, no. 3, pp. 30-34, 2019.

[24] Y. Liao and H. Zhou, "A systematic review of the English literature on system reform of universal higher education," Jiangsu Higher Education, vol. 1, pp. 7-13, 2021.

[25] Y. Mao, Q. Deng, and Z. Chen, "Parallel association rules incremental mining algorithm based on information entropy and genetic algorithm," Journal on Communications, vol. 42, no. 5, pp. 122-136, 2021.

[26] Z. Shi, "Returning to the ontology of education-a probe into the current reform of the education evaluation system in China," Educational Research, vol. 41, no. 9, pp. 4-15, 2020.

[27] A. Liang, Y. Ding, Q. Yan, and X. Liu, "Optimization of association rules algorithm in spark platform," Computer Engineering and Design, vol. 39, no. 12, pp. 3692-3699, 2018.

[28] J. Ahmad, O. K. Harsh, and A. Khan, "Performance optimization of higher education system by using secure \& reliable ERP system," International Journal of Recent Technology and Engineering, vol. 8, no. 5, pp. 1377-1384, 2020.

[29] A. A. Abdullaev, M. Atamirzayev, and K. F. Kholturayev, "Application of information resources and means in studying mathematical disciplines in universities," Journal of Critical Reviews, vol. 7, no. 5, pp. 1649-1651, 2020.

[30] J. Guo and Y. Gu, "Coordinated development of regional higher education in China: structural contradictions, cause analysis and strategies," China Higher Education Research, vol. 6, pp. 72-78, 2020.

[31] E. Akhmetshin, K. Barmuta, V. Vasilev, H. Okagbue, and O. Ijezie, "Principal directions of digital transformation of higher education system in sustainable education," E3S Web of Conferences, vol. 208, no. 1, pp. 9042-9061, 2020.

[32] L. Highman, "Remapping French higher education: towards a multi-tiered higher education system?" Tertiary Education and Management, vol. 26, no. 2, pp. 199-214, 2020.

[33] G. Luo, C. Tong, R. Wang, M. Xiao, and Y. Zhang, "Research on the application of virtual reality technology in higher education-a case study of operating system," The Chinese Journal of Ict in Education, vol. 12, pp. 37-42, 2021.

[34] D. I. Vasieva, "Modernization process in the higher education system," IJIE (Indonesian Journal of Informatics Education), vol. 2, no. 4, pp. 45-47, 2019.

[35] O. S. Ivanchenko, S. A. Tikhonovskova, A. A. Zalevskaya, and I. V. Belasheva, "Managing the professional and qualification and innovative potential of youth in the system of higher education," International Journal of Sociology and Social Policy, vol. 12, no. 1, pp. 252-273, 2020.
[36] J. Wang, "Challenges and solutions of higher education research," Modern University Education, vol. 3, pp. 1-11, 2020. 